\title{
Multitransitional observations of the CS core of L673
}

\author{
O. Morata, J. M. Girart, and R. Estalella
}

\author{
Departament d'Astronomia i Meteorologia, Universitat de Barcelona, Av. Diagonal, 647, 08028, Barcelona, Spain
}

Received 7 January 2002 / Accepted 29 July 2002

\begin{abstract}
A multitransitional study with the BIMA interferometric array was carried out toward the starless core found in the L673 region, in order to study the small-size structure of the cores detected with previous single-dish observations, which provides us with a test of the predictions of the chemical model of Taylor et al. $(1996,1998)$. We detected emission in the $\mathrm{CS}(J=2 \rightarrow 1), \mathrm{N}_{2} \mathrm{H}^{+}(J=1 \rightarrow 0)$, and $\mathrm{HCO}^{+}(J=1 \rightarrow 0)$ lines. Several clumps of size $\lesssim 0.08$ pc were found for each line distributed all over the region where previous single-dish emission was found (Morata et al. 1997). Each molecular transition traces differently the clump distribution, although in some cases the detected clumps are coincident. The distribution of the $\mathrm{N}_{2} \mathrm{H}^{+}$ emission and the single-dish $\mathrm{NH}_{3}$ emission are coincident and compatible with an origin in the same gas. The large fraction of missing flux measured for the CS $(2 \rightarrow 1)$ transition can be explained if the cloud is formed by a clumpy and heterogeneous medium. Four positions were selected to derive the abundance ratios $\left[\mathrm{N}_{2} \mathrm{H}^{+} / \mathrm{CS}\right]$ and $\left[\mathrm{HCO}^{+} / \mathrm{CS}\right]$ from the molecular column density determinations, and to compare them with the values predicted by the chemical model. The model was able to explain the interferometric observations, and, in particular, the chemical differentiation of the detected clumps and the coincidence of the $\mathrm{NH}_{3}$ and $\mathrm{N}_{2} \mathrm{H}^{+}$emissions. The lack of $\mathrm{HCO}^{+}$towards the two selected positions that trace the more evolved clumps cannot be accounted for by the model, but it is possibly due to strong self-absorption. We propose a classification of the studied clumps according to the stage of chemical evolution indicated by the molecular abundances.
\end{abstract}

Key words. molecular processes - ISM: abundances - ISM: clouds - ISM: molecules - ISM: individual objects: L673

\section{Introduction}

Low-mass star formation takes place in dense cores of molecular clouds (Beichman et al. 1986; Benson \& Myers 1989). The emission of several molecules, such as $\mathrm{CS}, \mathrm{NH}_{3}$, and $\mathrm{HCO}^{+}$, known to be good tracers of high density molecular gas, has been used to study these dense cores. However, very soon large discrepancies between the emission of these molecules were found in some sources (Zhou et al. 1989; Myers et al. 1991). To clarify the intrinsic differences between the emission of these molecules and how this emission is related to the actual distribution of the high density gas, we began a systematic comparison between the emission of the $\mathrm{CS}(J=1 \rightarrow 0)$ and $\mathrm{NH}_{3}$ $(J, K)=(1,1)$ transitions under similar conditions of angular resolution (Pastor et al. 1991; Morata et al. 1997). The comparison of the distribution of the $\mathrm{CS}$ and $\mathrm{NH}_{3}$ emission in 14 condensations of 11 star-forming regions confirmed the discrepancies. In particular, we found that there is a separation $\sim 0.2 \mathrm{pc}$ between the emission peaks of both molecules; regions traced by $\mathrm{CS}$ are larger than those traced by $\mathrm{NH}_{3}$; and $\mathrm{CS}$ lines are $\sim 0.5 \mathrm{~km} \mathrm{~s}^{-1}$ wider than those of $\mathrm{NH}_{3}$.

To explain these results, we developed a chemical model (Taylor et al. 1996) in which high density condensations, or dense cores, are formed by clumps $\lesssim 0.1 \mathrm{pc}$ in size, which

Send offprint requests to: $\mathrm{O}$. Morata,

e-mail: oscar@am.ub.es would be unresolved at moderate angular resolution observations such as the ones made in our study, of different mass, age, size and density. Most of the clumps disperse before $\mathrm{NH}_{3}$ abundances build up to significant levels, though these clumps contain substantial CS, so its emission should be observable. A few clumps, those sufficiently long lived, or being in a more advanced stage of physical and chemical evolution because of being denser or more massive, form a significant content of $\mathrm{NH}_{3}$, while CS abundance decreases with time. These clumps would possibly continue their evolution to eventually form stars. This model would account for the difference in size and separation between emission peaks of the $\mathrm{CS}$ and $\mathrm{NH}_{3}$ molecules.

Our chemical model explains the differences as a result of the speed at which the molecules form. Therefore, a classification could be made between "early-time" molecules and "late-time" molecules, according to the time at which these molecules reached their peak fractional abundance. We examined whether there were other potentially observable molecules that should show extended emission like CS, or more compact emission as $\mathrm{NH}_{3}$ (Taylor et al. 1998). Several candidates were found for both groups: e.g. $\mathrm{HCN}, \mathrm{H}_{2} \mathrm{CO}$ or $\mathrm{HC}_{3} \mathrm{~N}$ are found in the CS family, whilst $\mathrm{HCO}^{+}, \mathrm{SO}, \mathrm{NO}$ or $\mathrm{N}_{2} \mathrm{H}^{+}$are in the $\mathrm{NH}_{3}$ family.

In order to study the small-size structure of the cores detected with the single-dish observations, which also provides us with a test of the predictions of the chemical model, we 
Table 1. Transitions observed.

\begin{tabular}{|c|c|c|c|c|c|}
\hline Molecule & Transition & $\begin{array}{c}v \\
(\mathrm{GHz})\end{array}$ & $\begin{array}{c}\text { RMS } \\
\text { per channel } \\
\text { (Jy/beam) }\end{array}$ & $\begin{array}{l}\text { Spectral } \\
\text { resolution } \\
\left(\mathrm{km} \mathrm{s}^{-1}\right)\end{array}$ & Detected? \\
\hline $\mathrm{HCO}^{+}$ & $1 \rightarrow 0$ & 89.18852 & 0.17 & 0.33 & Yes \\
\hline $\mathrm{C}_{3} \mathrm{~S}$ & $16 \rightarrow 15$ & 92.48849 & 0.18 & 0.32 & No \\
\hline $\mathrm{N}_{2} \mathrm{H}^{+}$ & $1 \rightarrow 0$ & 93.17402 & 0.16 & 0.32 & Yes \\
\hline $\mathrm{C}_{2} \mathrm{~S}$ & $8_{7} \rightarrow 7_{6}$ & 93.87010 & 0.18 & 0.31 & No \\
\hline OCS & $8 \rightarrow 7$ & 97.30121 & 0.22 & 0.30 & No \\
\hline $\mathrm{CS}$ & $2 \rightarrow 1$ & 97.98097 & 0.18 & 0.30 & Yes \\
\hline HNCS & $9_{1,8} \rightarrow 8_{1,7}$ & 105.74386 & 0.25 & 0.28 & No \\
\hline $\mathrm{C}_{2} \mathrm{~S}$ & $9_{8} \rightarrow 8_{7}$ & 106.34774 & 0.24 & 0.28 & No \\
\hline $\mathrm{HC}_{3} \mathrm{~N}$ & $12 \rightarrow 11$ & 109.17363 & 0.26 & 0.27 & No \\
\hline SO & $2_{3} \rightarrow 1_{2}$ & 109.25218 & 0.25 & 0.27 & marginally \\
\hline OCS & $9 \rightarrow 8$ & 109.46306 & 0.25 & 0.27 & No \\
\hline
\end{tabular}

carried out high angular resolution observations towards L673 of several transitions corresponding to molecules of both families of species. The L673 high density condensation was previously detected in the CS $(J=1 \rightarrow 0)$ transition at moderate angular resolution by Morata et al. (1997). The L673 CS condensation is located at $\alpha(J 2000)=19^{\mathrm{h}} 20^{\mathrm{m}} 52^{\mathrm{s}} .1, \delta(J 2000)=$ $+11^{\circ} 15^{\prime} 29^{\prime}$. 5 , at $\sim 9$ '.5 to the southeast of IRAS $19180+1116$. This condensation is well suited to our purposes for several reasons. A high density condensation has been detected nearby in the $\mathrm{NH}_{3}(J, K)=(1,1)$ transition by Sepúlveda et al. (2002), which shows the same general behavior found in the other sources of our survey: a separation from the CS emission peak $\sim 2^{\prime}$, and a $\mathrm{NH}_{3}$ condensation size clearly smaller than the CS condensation size. It is located nearby, the estimated distance to the L673 cloud is $\sim 300$ pc (Herbig \& Jones 1983), which helps in the study of the smaller size structure of the cloud. Finally, the condensations detected in $\mathrm{CS}$ and $\mathrm{NH}_{3}$ show no signs of tracers of star formation, such as infrared or radio continuum sources, Herbig-Haro objects or molecular outflows, which indicates that it is probably a quiescent core, maybe in the first stages of collapse, before forming a stellar core or a Class 0 star, and thus fulfilling the conditions of the chemical model we developed.

\section{Observations}

The observations of the L673 region were carried out in 1998 May with the 10-antenna BIMA array ${ }^{1}$ at the Hat Creek Radio Observatory in the $\mathrm{C}$ configuration. The phase calibrators were $1751+096$ and 3C395. In order to include the positions of the $\mathrm{CS}(J=1 \rightarrow 0)$ and $\mathrm{NH}_{3}(J, K)=(1,1)$ transitions singledish emission peaks of the maps by Morata et al. (1997) and Sepúlveda et al. (2002), we made a two-point mosaic with each of the fields centered approximately at the position of these two peaks. Thus, one of the fields was centered at the position $\alpha(2000)=19^{\mathrm{h}} 20^{\mathrm{m}} 52^{\mathrm{s}} .2$, and $\delta(2000)=+11^{\circ} 13^{\prime} 57^{\prime \prime}$, and the other located $90^{\prime \prime}$ to the North. Three frequency

\footnotetext{
1 The BIMA array is operated by the Berkeley-Illinois-Maryland Association with support from the National Science Foundation.
}

setups were used, centered at 91, 96 and $107 \mathrm{GHz}$. The digital correlator was configured to observe simultaneously several molecular line transitions at moderately high spectral resolution, $\sim 0.3 \mathrm{~km} \mathrm{~s}^{-1}$. The target molecular lines were $\mathrm{HCO}^{+}$ $(J=1 \rightarrow 0), \mathrm{N}_{2} \mathrm{H}^{+}(J=1 \rightarrow 0), \mathrm{CS}(J=2 \rightarrow 1)$, SO $\left(J_{K}=\right.$ $\left.2_{3} \rightarrow 1_{2}\right)$ and $\mathrm{C}^{18} \mathrm{O}(J=1 \rightarrow 0)$. System temperatures for the 91,96 and $107 \mathrm{GHz}$ setups were in the $180-500 \mathrm{~K}, 250-700 \mathrm{~K}$ and $500-1000 \mathrm{~K}$ range, respectively. The calibration and data reduction were performed using the MIRIAD software package (Sault et al. 1995). Mosaic maps were done with the visibility data weighted by the associated system temperatures and using natural weighting, and applying the primary beam correction. The resulting synthesized beam for the 91,96 and $107 \mathrm{GHz}$ setups were $12^{\prime \prime} .7 \times 9$ '. $^{\prime}, \mathrm{PA}=-14^{\circ} ; 13^{\prime \prime} .3 \times 10^{\prime} .5, \mathrm{PA}=-17^{\circ}$; and $13^{\prime \prime} 1 \times 9$ ! $3, \mathrm{PA}=-8^{\circ}$, respectively. The transitions of all the observed lines, their velocity resolution and the achieved rms noise with this velocity resolution are listed in Table 1 . For the continuum emission we used the data from the $96 \mathrm{GHz}$ correlator setup, which provided a bandwidth of $800 \mathrm{MHz}$ centered at the frequency of $95.9 \mathrm{GHz}$. No emission was detected at above $8 \mathrm{mJy} \mathrm{beam}^{-1}$ (at a 3- $\sigma$ level).

\section{Results}

\subsection{Morphology of the molecular emission}

We detected emission in three of the transitions: CS $(J=2 \rightarrow 1), \mathrm{N}_{2} \mathrm{H}^{+}(J=1 \rightarrow 0)$, and $\operatorname{HCO}^{+}(J=1 \rightarrow 0)$. We detected marginally, at $\sim 2 \sigma$ level, the $\mathrm{SO}\left(2_{3} \rightarrow 1_{2}\right)$ transition. The remaining transitions of Table 1 , which where observed mainly because they were found inside the frequency range of the observations, were not detected. It must be noted that all these undetected transitions have an upper level energy with temperatures higher than $15 \mathrm{~K}$, and most of them, OCS is the exception, have also a high dipole moment. Thus, the temperature and density conditions of the L673 core, and the low abundances expected for these molecules make them very difficult to detect.

Figures 1 to 3 show the maps of the zero-order moment (integrated intensity) of the emission of the detected molecules. 


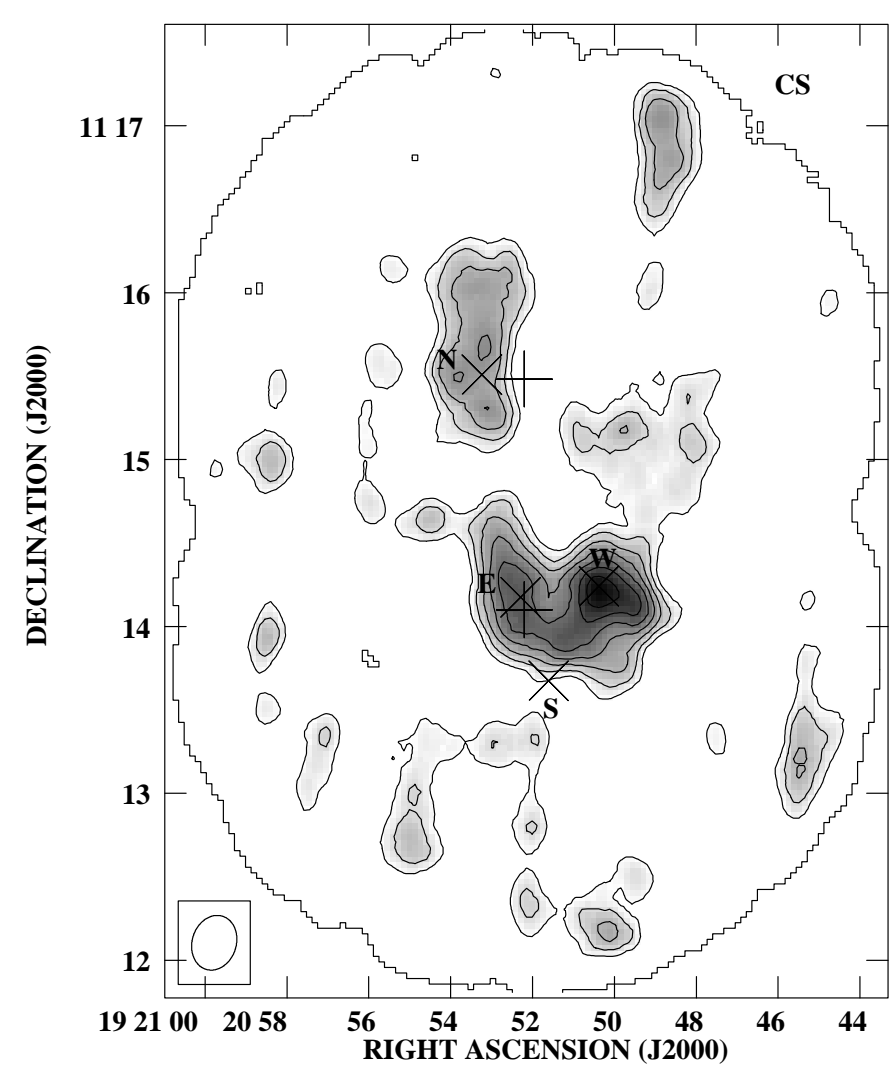

Fig. 1. Integrated emission of the CS $(J=2 \rightarrow 1)$ line for the $V_{\mathrm{LSR}}$ range 5.6-9.8 $\mathrm{km} \mathrm{s}^{-1}$. Contours are 0.96, 1.20, 1.44, 1.68, 1.92, 2.16, 2.40, $2.64 \mathrm{Jy} \mathrm{beam}^{-1} \mathrm{~km} \mathrm{~s}^{-1}$. The beam $\left(20^{\prime \prime} \times 15^{\prime \prime} 8\right)$ is shown in the bottom left-hand corner. The upright crosses indicate the nominal position of the single-dish emission peak of the CS $(J=1 \rightarrow 0)$ line (north) and $\mathrm{NH}_{3}(J, K)=(1,1)$ line (south). The tilted crosses indicate the position of the four points selected for further study (see Table 2). The bordering contour indicates the two fields observed.

These maps were obtained after convolving the original maps with a Gaussian function, with a resulting beam of $\sim 20^{\prime \prime} \times 15^{\prime \prime}$, in order to make a more meaningful comparison between the emission of each molecule.

A clumpy distribution of the emission is shown in the integrated intensity map of the CS $(J=2 \rightarrow 1)$ transition (Fig. 1). The more intense emission is found in two clumps near the position of the single-dish $\mathrm{NH}_{3}(J, K)=(1,1)$ emission peak: one almost coinciding with its nominal position, and the other peaking $\sim 30^{\prime \prime}$ to the west. Weaker emission is found in a clump near the position of the single-dish CS $(J=1 \rightarrow 0)$ emission maximum, $\sim 1.5$ to the north.

Figure 2 shows the integrated intensity map of $\mathrm{N}_{2} \mathrm{H}^{+}$ $(J=1 \rightarrow 0)$. Two clumps of emission are found, one almost coincident with the nominal position of the $\mathrm{NH}_{3}(J, K)=(1,1)$ emission peak, and the other, more intense, $\sim 0.6$ to the south. No emission is detected around the position of the CS $(J=1 \rightarrow 0)$ single dish emission peak.

The integrated intensity map of the $\operatorname{HCO}^{+}(J=1 \rightarrow 0)$ emission (Fig. 3) shows several isolated emission enhancements distributed around the positions of the $\mathrm{CS}$ and $\mathrm{NH}_{3}$ single-dish emission peaks. The most intense emission tends to be around the position of the $\operatorname{CS}(J=1 \rightarrow 0)$ emission peak

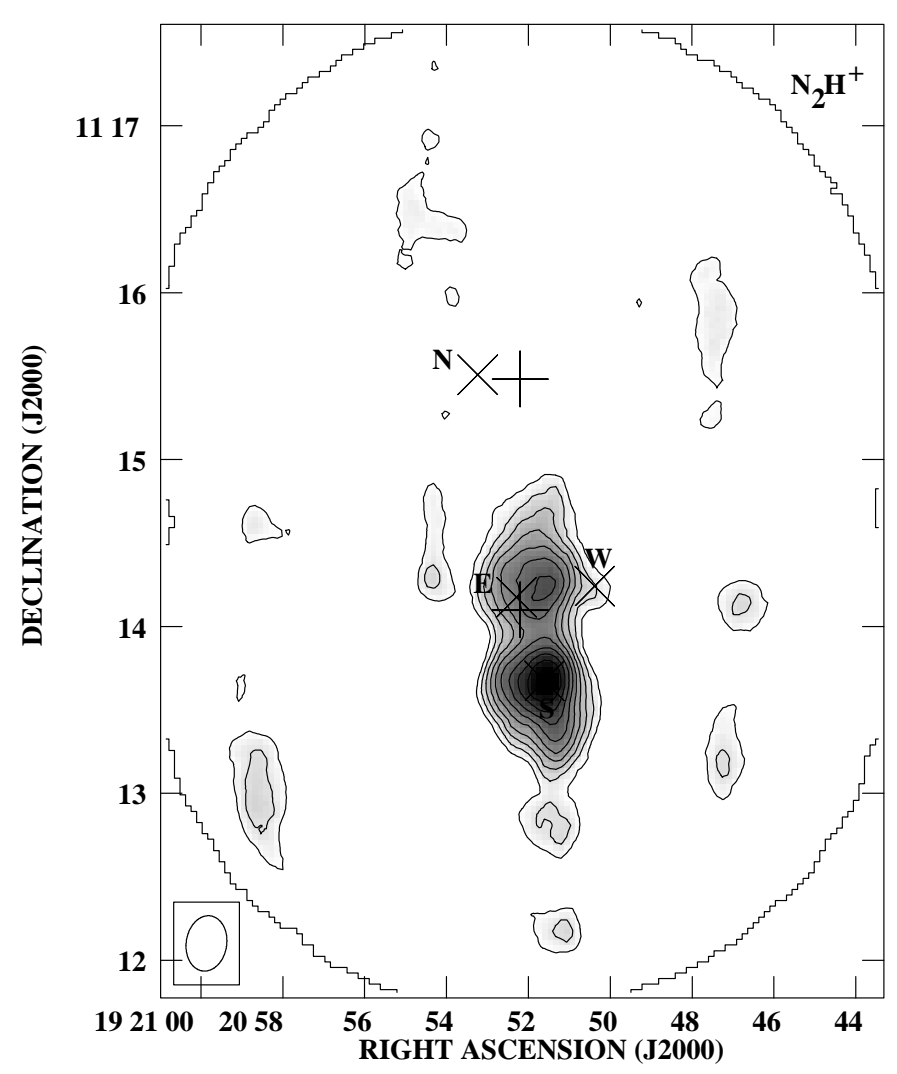

Fig. 2. Same as in Fig. 1 for the $\mathrm{N}_{2} \mathrm{H}^{+}(J=1 \rightarrow 0)$ line for the $V_{L S R}$ range $-1.4-14.2 \mathrm{~km} \mathrm{~s}^{-1}$. Contours are 2.55, 3.06, 3.57, 4.08, 4.59, $5.10,5.61,6.12,6.63,7.14 \mathrm{Jy}_{\text {beam }}{ }^{-1} \mathrm{~km} \mathrm{~s}^{-1}$. The beam $\left(20^{\prime \prime} \times 14^{\prime \prime} 6\right)$ is shown in the bottom left-hand corner.

or in-between the two peaks, with a N-S elongation. Another intense enhancement is found $\sim 1$ ! 1 south-east of the $\mathrm{NH}_{3}$ emission peak nominal position.

Comparing the integrated intensity maps of the three molecules, we found that $\mathrm{N}_{2} \mathrm{H}^{+}$emission was more concentrated than $\mathrm{CS}$ and $\mathrm{HCO}^{+}$emission, which were found more spread all over the region. CS $(J=2 \rightarrow 1)$ emission in the southern region coincided with the northern clump of $\mathrm{N}_{2} \mathrm{H}^{+}$ emission. Moreover, the northern $\mathrm{N}_{2} \mathrm{H}^{+}$emission peak was found between the two CS $(J=2 \rightarrow 1)$ local emission enhancements. $\mathrm{CS}(J=2 \rightarrow 1)$ and $\mathrm{HCO}^{+}$emission were found to coincide closely at the northern part of the region, especially around the CS $(J=1 \rightarrow 0)$ emission peak. On the contrary, $\mathrm{N}_{2} \mathrm{H}^{+}$and $\mathrm{HCO}^{+}$emission coincided only marginally in general. Finally, we were also able to find an emission enhancement in the marginally detected SO emission very near the position of the $\mathrm{N}_{2} \mathrm{H}^{+}$northern clump.

Table 2 lists the four positions we selected in the mapped region in order to compare the physical parameters of the gas traced by each molecule. The selected positions were related to the most prominent higher resolution clumps found in our observations, and corresponded to local intensity peaks in the emission of the detected molecules. The four positions were labeled as S, E, W and N, and roughly correspond to the South peak position of the $\mathrm{N}_{2} \mathrm{H}^{+}$integrated intensity map, the East CS local maximum, the West CS maximum, and the North 
Table 2. Selected positions.

\begin{tabular}{lcrl}
\hline \hline & \multicolumn{2}{c}{ Position } & map counterpart \\
\cline { 2 - 3 } & $\alpha(\mathrm{J} 2000)$ & $\delta(\mathrm{J} 2000)$ & \\
\hline South (S) & $19^{\mathrm{h}} 20^{\mathrm{m}} 51.61^{\mathrm{s}}$ & $11^{\circ} 13^{\prime} 40^{\prime} 5$ & \multirow{2}{*}{$\mathrm{N}_{2} \mathrm{H}^{+}(J=1 \rightarrow 0)$ south peak } \\
East (E) & $19^{\mathrm{h}} 20^{\mathrm{m}} 52.29^{\mathrm{s}}$ & $11^{\circ} 14^{\prime} 10^{\prime} 5$ & $\mathrm{CS}(J=2 \rightarrow 1)$ eastern peak \\
West (W) & $19^{\mathrm{h}} 20^{\mathrm{m}} 50.38^{\mathrm{s}}$ & $11^{\circ} 14^{\prime} 14^{\prime \prime} 5$ & $\mathrm{CS}(J=2 \rightarrow 1)$ western peak \\
North (N) & $19^{\mathrm{h}} 20^{\mathrm{m}} 53.24^{\mathrm{s}}$ & $11^{\circ} 15^{\prime} 30^{\prime} 5$ & $\mathrm{HCO}^{+}(J=1 \rightarrow 0)$ NE enhancement \\
\hline
\end{tabular}

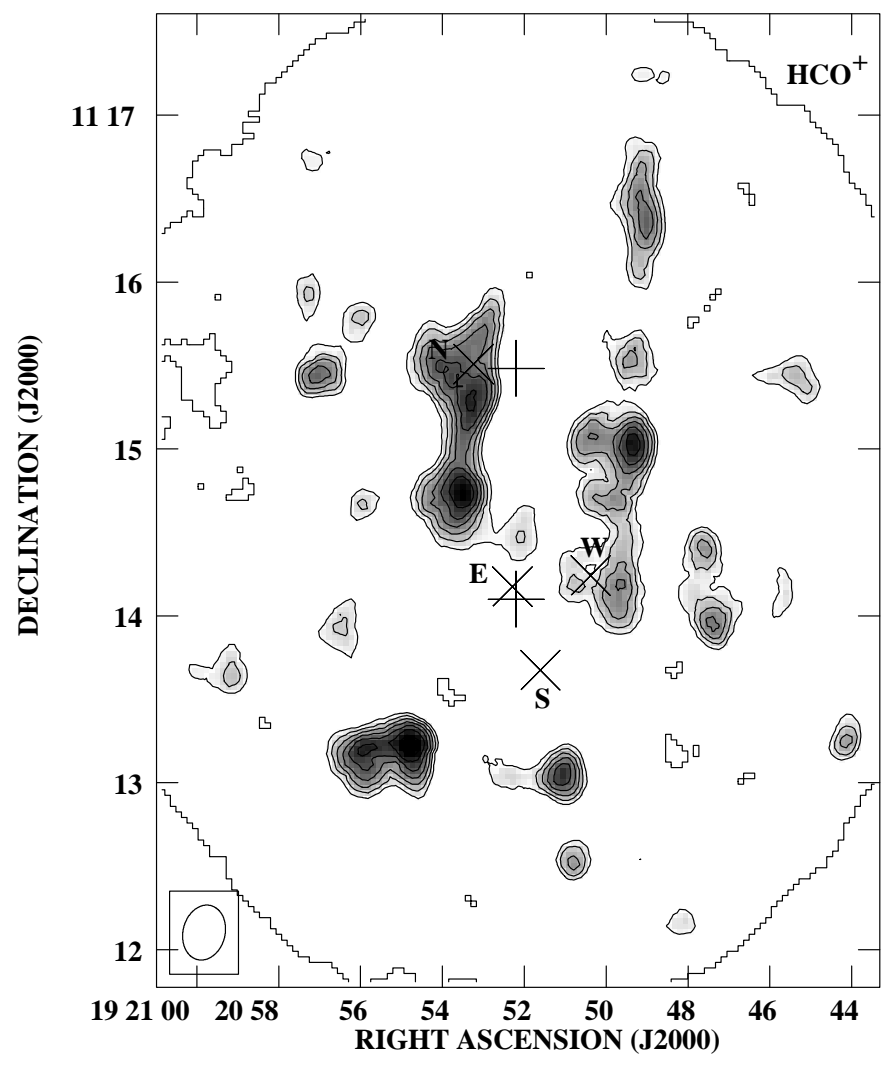

Fig. 3. Same as in Fig. 1 for the $\mathrm{HCO}^{+}(J=1 \rightarrow 0)$ line for the $V_{\mathrm{LSR}}$ range 5.6-9.8 $\mathrm{km} \mathrm{s}^{-1}$. Contours are $0.96,1.08,1.20,1.32,1.44,1.56$, $1.68 \mathrm{Jy} \mathrm{beam}^{-1} \mathrm{~km} \mathrm{~s}^{-1}$. The beam $\left(20^{\prime \prime} \times 15^{\prime \prime} .1\right)$ is shown in the bottom left-hand corner.

enhancement in the $\mathrm{HCO}^{+}$emission near the $\mathrm{CS}(J=1 \rightarrow 0)$ emission peak, respectively.

\subsection{Kinematic structure}

Figure 4 shows the spectra obtained for the three transitions detected in the region in the four selected points. Table 3 lists the fitted line parameters for each detected transition. CS emission is detected, with varying intensity, in all four positions, whereas $\mathrm{HCO}^{+}$emission is only detected at the $\mathrm{N}$ and $\mathrm{W}$ positions, and $\mathrm{N}_{2} \mathrm{H}^{+}$emission at the $\mathrm{S}$ and $\mathrm{E}$ positions. Line center velocities for $\mathrm{CS}$ and $\mathrm{HCO}^{+}$at the positions where a reliable fit could be obtained are compatible with being originated in the same bulk of gas. The difference in the line center velocity is $\sim 0.3-0.5 \mathrm{~km} \mathrm{~s}^{-1}$, which is the velocity resolution of our observations. However, line center velocities for the $\mathrm{N}_{2} \mathrm{H}^{+}$

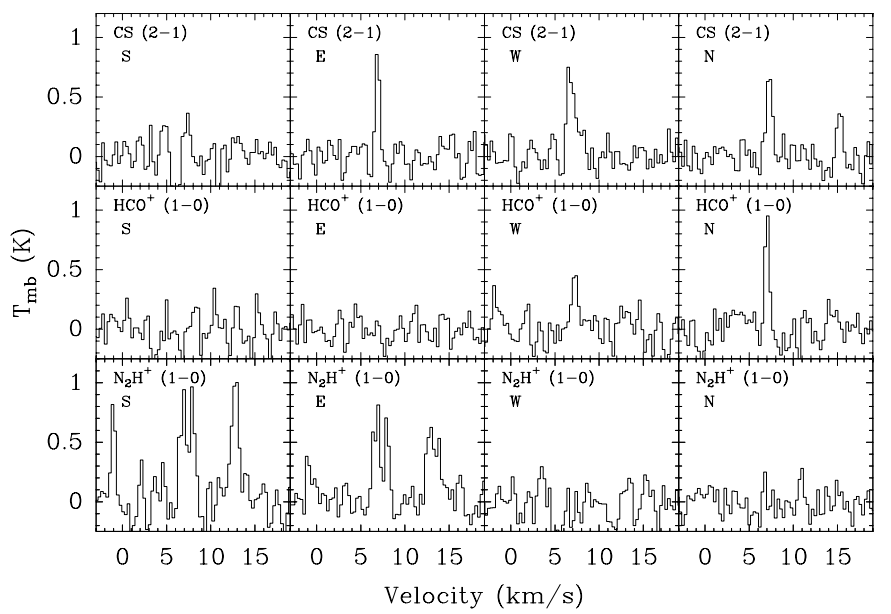

Fig. 4. Spectra of the CS $(J=2 \rightarrow 1), \mathrm{HCO}^{+}(J=1 \rightarrow 0)$, and $\mathrm{N}_{2} \mathrm{H}^{+}$ $(J=1 \rightarrow 0)$ transitions at the four selected positions in our mapped region.

spectra were shifted $>0.7 \mathrm{~km} \mathrm{~s}^{-1}$ from the CS line velocities. Using the much better determined frequency for the main $\mathrm{N}_{2} \mathrm{H}^{+}$ $(J=1 \rightarrow 0)$ component $\left(F_{1}, F=2,3 \rightarrow 1,2\right)$ given by Caselli et al. (1995): $93.17378 \mathrm{GHz}$, we found a good agreement with CS spectra line velocities, $\lesssim 0.3 \mathrm{~km} \mathrm{~s}^{-1}$. We have corrected the $\mathrm{N}_{2} \mathrm{H}^{+}$line velocities accordingly, as Table 3 shows. Linewidths are $<0.8 \mathrm{~km} \mathrm{~s}^{-1}$, very similar to the observed values in the single-dish observations, except for the $\mathrm{W}$ clump where velocity dispersion is higher, $\sim 1.0 \mathrm{~km} \mathrm{~s}^{-1}$.

\subsection{Physical parameters}

We were able to estimate the excitation temperature for the $\mathrm{N}_{2} \mathrm{H}^{+}$data at the two positions, $\mathrm{S}$ and $\mathrm{E}$, where we had good signal-to-noise spectra. The values obtained at both positions are $\sim 4 \mathrm{~K}$ (assuming a beam filling factor $f=1$ ), which is compatible with the value obtained from the CS $(J=1 \rightarrow 0)$ observations, 4.2 K (Morata et al. 1997), and slightly lower than the value obtained from the $\mathrm{NH}_{3}(J, K)=(1,1)$ observations, 5.7 K (Sepúlveda et al. 2002). The total line opacity of the $\mathrm{N}_{2} \mathrm{H}^{+}(J=1 \rightarrow 0)$ transition at both positions is $\sim 1.0$. We estimated an upper limit for the beam averaged column density using the value for the excitation temperature obtained from the $\mathrm{CS}(J=1 \rightarrow 0)$ and $\mathrm{N}_{2} \mathrm{H}^{+}(J=1 \rightarrow 0)$ observations, $T_{\text {ex }}=4 \mathrm{~K}$, and assuming a filling-factor $f=1$. However, the filling-factor could be $f<1$. In this case, the excitation temperature would be greater than $4 \mathrm{~K}$, but likely less than $10 \mathrm{~K}$, 
Table 3. CS $(J=2 \rightarrow 1), \mathrm{HCO}^{+}(J=1 \rightarrow 0)$, and $\mathrm{N}_{2} \mathrm{H}^{+}(J=1 \rightarrow 0)$ lines and physical parameters obtained at the four selected positions in our region.

\begin{tabular}{lllllllll}
\hline $\begin{array}{l}\text { Molecule \& } \\
\text { Transition }\end{array}$ & Pos. & $\begin{array}{l}T_{\mathrm{mb}} \\
(\mathrm{K})\end{array}$ & $\begin{array}{l}A \tau_{m}{ }^{(a)} \\
(\mathrm{K})\end{array}$ & $\begin{array}{l}V_{\mathrm{LSR}} \\
\left(\mathrm{km} \mathrm{s}^{-1}\right)\end{array}$ & $\begin{array}{l}\Delta V \\
\left(\mathrm{~km} \mathrm{~s}^{-1}\right)\end{array}$ & $\tau_{m}{ }^{(b)}$ & \multicolumn{1}{c}{$\begin{array}{l}N_{\mathrm{mb}}{ }^{(c)} \\
\left(10^{11} \mathrm{~cm}^{-2}\right)\end{array}$} \\
\hline $\mathrm{CS}$ & $\mathrm{S}$ & $0.35 \pm 0.12$ & & $+7.4 \pm 0.1$ & $0.7 \pm 0.2$ & & $0.05-0.4^{(d)}$ & $12.0-26.9$ \\
$(J=2 \rightarrow 1)$ & $\mathrm{E}$ & $0.96 \pm 0.11$ & & $+6.9 \pm 0.1$ & $0.5 \pm 0.1$ & & $0.15-2.1^{(d)}$ & $25.3-84.8$ \\
& $\mathrm{~W}$ & $0.73 \pm 0.10$ & & $+6.8 \pm 0.1$ & $1.0 \pm 0.1$ & & $0.11-1.1^{(d)}$ & $37.2-99.6$ \\
& $\mathrm{~N}$ & $0.72 \pm 0.11$ & & $+7.3 \pm 0.1$ & $0.8 \pm 0.1$ & & $0.11-1.1^{(d)}$ & $27.1-72.2$ \\
\hline $\mathrm{HCO}^{+}$ & $\mathrm{S}$ & $<0.37^{(e)}$ & & - & - & & $<0.39^{(d)}$ & $<4.73$ \\
$(J=1 \rightarrow 0)$ & $\mathrm{E}$ & $<0.29^{(e)}$ & & - & - & & $<0.29^{(d)}$ & $<3.60$ \\
& $\mathrm{~W}$ & $0.48 \pm 0.13$ & & $+7.2 \pm 0.1$ & $0.8 \pm 0.3$ & & $0.07-0.6^{(d)}$ & $4.7-7.7$ \\
& $\mathrm{~N}$ & $1.02 \pm 0.12$ & & $+7.0 \pm 0.1$ & $0.6 \pm 0.1$ & & $0.16-2.3^{(d)}$ & $7.7-19.1$ \\
\hline $\mathrm{N}_{2} \mathrm{H}^{+}$ & $\mathrm{S}$ & & $0.32 \pm 0.05$ & $+7.0 \pm 0.1$ & $0.5 \pm 0.1^{(f)}$ & $0.25 \pm 0.10$ & $1.0^{(g)}$ & $2.2-3.1$ \\
$(J=1 \rightarrow 0)$ & $\mathrm{E}$ & & $0.24 \pm 0.04$ & $+7.0 \pm 0.1$ & $0.5 \pm 0.1^{(f)}$ & $0.27 \pm 0.10$ & $1.0^{(g)}$ & $1.7-2.4$ \\
& $\mathrm{~W}$ & & $<0.20^{(e)}$ & - & - & - & $<0.7^{(d)}$ & $<2.0$ \\
& $\mathrm{~N}$ & & $<0.16^{(e)}$ & - & - & - & $<0.5^{(d)}$ & $<1.6$ \\
\hline
\end{tabular}

(a) Derived from the transfer equation, where $A=f\left[J\left(T_{\mathrm{ex}}\right)-J\left(T_{\mathrm{bg}}\right)\right]$ is the "amplitude" (Pauls et al. 1983), $f$ is the filling factor, $T_{\mathrm{ex}}$ the excitation temperature of the transition, $T_{\mathrm{bg}}$ the background radiation temperature, and $J(T)$ the intensity in units of temperature.

(b) Optical depth of the main line.

(c) Beam averaged column density calculated from

$$
N_{\mathrm{mb}}=f N=\frac{3 k}{4 \pi^{3} v \mu_{D}^{2}} \frac{T_{\mathrm{ex}}+\frac{h v}{6 J k}}{\exp \left[-h v(J-1) / 2 k T_{\mathrm{ex}}\right]-\exp \left[-h v(J+1) / 2 k T_{\mathrm{ex}}\right]} \frac{1}{J\left(T_{\mathrm{ex}}\right)-J\left(T_{\mathrm{bg}}\right)} \int T_{\mathrm{mb}} d V
$$

where $f$ is the beam filling factor, $\mu_{D}$ the dipole moment of the molecule, $J$ the lower level of the transition, and $J(T)$ the intensity in units of temperature.

(d) Derived from the transfer equation, assuming for the excitation temperature upper and lower values of $10 \mathrm{~K}$ and $4 \mathrm{~K}$, respectively.

(e) Upper limit taken as $3 \sigma$, where $\sigma$ is the sensitivity per channel.

$(f)$ Intrinsic line width

(g) Total line opacity

because the kinetic temperature in low-mass dense cores is typically about $10 \mathrm{~K}$, and the excitation temperature will not be greater than this value. Thus, we used an upper value for the excitation temperature $T_{\text {ex }}=10 \mathrm{~K}$, which implies a lower limit for the beam average column density. The values for the line opacity, $\tau$, and the beam averaged column density, $N_{\mathrm{mb}}$ for the four selected positions are shown in Table 3.

\subsection{Comparison with single-dish observations}

We compared the CS $(J=2 \rightarrow 1)$ interferometric and $(J=1 \rightarrow 0)$ single-dish emissions. Figure 5 shows the singledish CS $(J=1 \rightarrow 0)$ spectra obtained at the positions of the $\mathrm{CS}(J=1 \rightarrow 0)$ and $\mathrm{NH}_{3}(J, K)=(1,1)$ emission peaks, compared with the BIMA CS $(J=2 \rightarrow 1)$ spectra obtained at the same positions after convolving the data with a Gaussian function to obtain a 1.9 resulting beam, equal to the beamsize of the single-dish observations. Line center velocities agree within $0.3 \mathrm{~km} \mathrm{~s}^{-1}$, our spectral resolution in both sets of observations. Line widths are almost coincident at the southern position, while the $(J=1 \rightarrow 0)$ line is $\sim 0.35 \mathrm{~km} \mathrm{~s}^{-1}$ wider at the northern position. In order to estimate the flux loss in our interferometric observations, we calculated the line intensity we should obtain for the $(J=2 \rightarrow 1)$ line from the $(J=1 \rightarrow 0)$ line opacity and excitation temperature obtained previously in the single-dish observations (Morata et al. 1997). We found that the measured line intensity is $\sim 8 \%$ in the northern position, and $\sim 12 \%$ in the south position from the estimated values. These results are compatible with the characteristics of the BIMA array, which is insensitive to structures larger than about 10 times the synthesized beamwidth (Wright 1996), which in our case is $\sim 1$ '.5-2' $(0.13-0.17 \mathrm{pc})$, of the order of the single-dish beamsize.

However, the existence of such an extended component, responsible for $\sim 90 \%$ of the single-dish line intensity, would not affect significantly the beam averaged column densities determined from our interferometric observations. Assuming a size of $120^{\prime \prime}$ for the extended component, its contribution to the beam averaged column density for a beam size of $17^{\prime \prime}$ would be of the order of $90 \% \times\left(17^{\prime \prime} / 120^{\prime \prime}\right)^{2}=2 \%$. Thus, the beam averaged column densities determined from our observations are not significantly affected by the missing emission by the BIMA interferometer, assuming that it is due to an extended cloud component.

Figure 6 shows the distribution of the single-dish CS $(J=1 \rightarrow 0)$ (Morata et al. 1997) and $\mathrm{NH}_{3}(J, K)=(1,1)$ (Sepúlveda et al. 2002) emission in the same region mapped by the interferometric observations. We found that most of the strongest CS $(J=2 \rightarrow 1)$ emission and particularly all the 


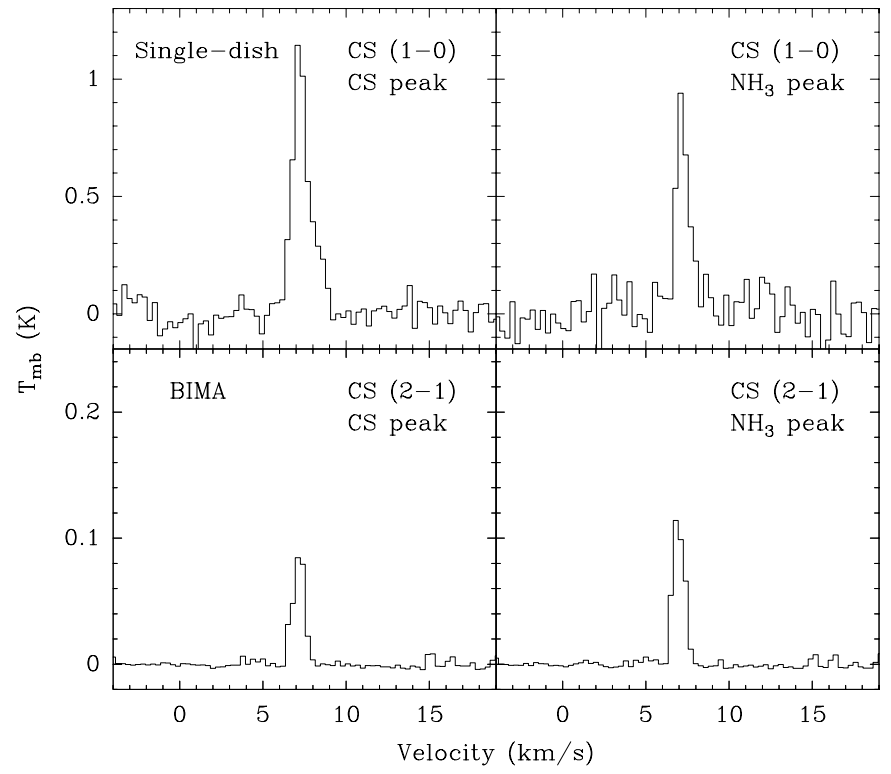

Fig. 5. Spectra of the CS $(J=1 \rightarrow 0)$ transitions obtained at the positions of the $\mathrm{CS}(J=1 \rightarrow 0)$ and $\mathrm{NH}_{3}(J, K)=(1,1)$ single-dish emission peaks with the Yebes telescope (top), compared with the spectra of the CS $(J=2 \rightarrow 1)$ transition obtained at the same positions after convolving the BIMA data with a beam of 1'.9 equal to the single-dish beam (bottom). Note the different vertical scales.

detected $\mathrm{N}_{2} \mathrm{H}^{+}$emission were enclosed inside the halfmaximum contours of the $\mathrm{NH}_{3}$ map, located at the southern region of the map. All four relative emission enhancements in these two transitions are also enclosed inside the beam of the single-dish observations. $\mathrm{HCO}^{+}$emission was fainter around this southern region, and marginal in the eastern, western and southern margins of the $\mathrm{NH}_{3}$ intensity contours. Around the northern region, no $\mathrm{N}_{2} \mathrm{H}^{+}$emission was detected, but $\mathrm{CS}$ and $\mathrm{HCO}^{+}$emission were enclosed by the beam of the CS singledish observations. Emission around this position was not as concentrated as around the southern region, but we found intense $\mathrm{HCO}^{+}$and $\mathrm{CS}(J=2 \rightarrow 1)$ emission very near the beam center.

\section{Discussion}

\subsection{Structure of the core}

The integrated intensity maps of the CS $(J=2 \rightarrow 1)$, $\mathrm{N}_{2} \mathrm{H}^{+}(J=1 \rightarrow 0)$, and $\mathrm{HCO}^{+}(J=1 \rightarrow 0)$ transitions obtained with the BIMA interferometer showed that a much clumpier medium is revealed by the high angular resolution observations than by the single-dish maps of CS $(J=1 \rightarrow 0)$ (Morata et al. 1997) and $\mathrm{NH}_{3}(J, K)=(1,1)$ (Sepúlveda et al. 2002 ) obtained with an angular resolution of $\sim 1 ' 5$. Several clumps of size $\lesssim 0.08 \mathrm{pc}$ are found distributed all over the region where the strongest emission of the single-dish observations was located, which would support the idea that at least part of the single-dish emission was originated in clumps of smaller size, $<0.1 \mathrm{pc}$ (Taylor et al. 1996). Moreover, these small size clumps, although coinciding in some cases, are traced differently by each molecule, which would support the idea of a

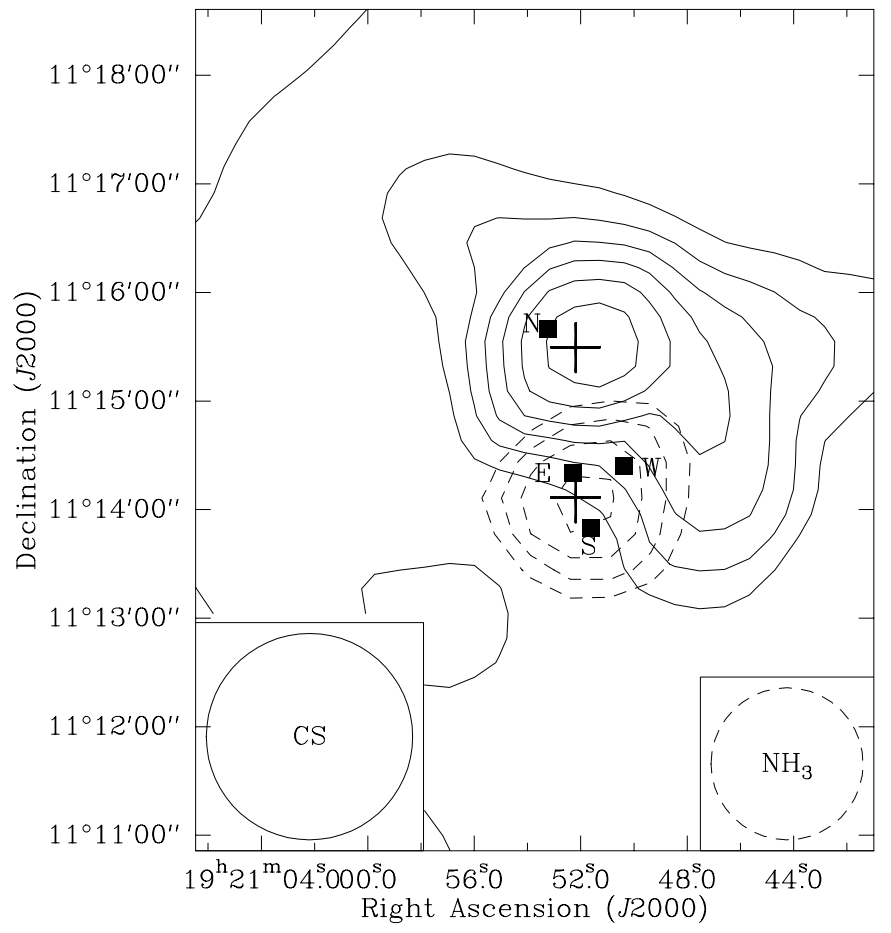

Fig. 6. Contour map of the single-dish integrated line intensity of the CS $(J=1 \rightarrow 0)$ line (solid line) obtained with the Yebes telescope (Morata et al. 1997), and the single-dish main beam brightness temperature of the $\mathrm{NH}_{3}(J, K)=(1,1)$ transition (dashed line) obtained with the Haystack telescope (Sepúlveda et al. 2002). The lowest CS contour level is $0.48 \mathrm{~K} \mathrm{~km} \mathrm{~s}^{-1}$, and the increment is $0.05 \mathrm{~K} \mathrm{~km} \mathrm{~s}^{-1}$. The lowest $\mathrm{NH}_{3}$ contour level is $1.35 \mathrm{~K}$, and the increment is $0.3 \mathrm{~K}$. The crosses indicate the nominal position of the single-dish emission peaks of the CS $(J=1 \rightarrow 0)$ line (north) and $\mathrm{NH}_{3}(J, K)=(1,1)$ line (south). The filled squares indicate the position of the four points selected for further study in the interferometric observations.

chemical differentiation between each clump of gas depending on the local density and age. However, we found that $\sim 90 \%$ of the single-dish CS emission is not detected by the interferometric observations. As we have already pointed out, the BIMA array is insensitive to structures larger than $\sim 0.17 \mathrm{pc}$, which could mean that part of the emission could come from chemically young clumps of that size or larger, undetectable then by BIMA, but whose emission was detected by the singledish observations. Another possibility is that there could also be clumps smaller than the ones we found, of low density, and with emission not strong enough to be detected with the interferometer.

In order to qualitatively test which of the aforementioned two possibilities is more consistent with our observations, we modeled the filtering effect of the BIMA interferometer response to a molecular core with an homogeneous and extended geometry and a clumpy heterogeneous geometry. The process of generating synthetic visibility data matching that expected from true observations with the BIMA interferometer was performed by using the MIRIAD task UVGEN. This process is described by Girart \& Acord (2001). Our synthetic observations had the same phase center as the L673 observations and were done for the BIMA C-array configuration. The total flux 
adopted for both cases, the extended and clumpy structures, was similar to that expected for the CS $(J=2 \rightarrow 1)$ transition, calculated from the measured CS $(J=1 \rightarrow 0)$ intensity. The results obtained were:

1. Extended and homogeneous medium. The presence of the extended emission was modeled as single large component. We tested the filtering effect of the interferometer for different sizes and different center positions of the cloud with respect to the phase center. We found that in order to achieve a missing flux of about $90 \%$, the value estimated for the BIMA observations, large sizes were required: diameter $\gtrsim 2^{\prime}$. The resulting synthetic maps showed strong negative lobes extending $\sim 40^{\prime \prime}$ along the $\mathrm{N}-\mathrm{S}$ direction, at both sides of the center of the synthetic cloud. This was due to a combination of the effects of source structure, with a large fraction of undetected flux by the interferometer, and visibility coverage of the BIMA interferometer for a source of low declination $\left(\delta=11^{\circ}\right.$ for L673). These negative structures could not be removed in the cleaning process. The cleaned maps had absolute peak intensities of the negative structures with similar, or even higher, values than the positive structures.

However the real BIMA CS $(J=2 \rightarrow 1)$ channel maps does not show such strong negative structures. At the central velocities of the CS line there are some negative lobes, but their intensity is only a $\$ 40 \%$ of the positive peaks.

2. Clumpy and heterogeneous medium. The chemical models of Taylor et al. $(1996,1998)$ assume that the clouds are formed by clumps of $\lesssim 0.1 \mathrm{pc}$ with different sizes, ages and masses. From this assumption it is expected a cloud formed with only few massive clumps and several clumps less massive. So, we adopted a simplistic model of a cloud formed with one massive, thereby strong, clump and between 15 and 20 less massive, thereby weaker, clumps. We found that even most of the emitting flux came from the smaller clumps, the BIMA synthetic resulting maps were clearly dominated by the strongest clump, and a large fraction of the emission from the weaker clumps was undetected by the interferometer. In some of the cases modeled, the total missing flux was of $\sim 80 \%$ of the total flux. The residual negative structures of the synthetic maps were $30-50 \%$ of the positive.

In summary, without going to an exhaustive analysis of the response of the BIMA interferometer to the different cloud structures, we find that in L673 the missing flux is most probably due to a clumpy heterogeneous structure.

\subsection{Chemical abundances}

In our maps, we found that all the detected $\mathrm{N}_{2} \mathrm{H}^{+}$emission was enclosed inside the half-maximum contours of the $\mathrm{NH}_{3}$ map of Sepúlveda et al. (2002), with the strongest $\mathrm{N}_{2} \mathrm{H}^{+}$emission found inside the highest $\mathrm{NH}_{3}$ contours. The nominal position of the $\mathrm{NH}_{3}$ emission peak is placed between the two local $\mathrm{N}_{2} \mathrm{H}^{+}$emission peaks, although the beam enclosed both maxima. The chemical model developed by Taylor et al. (1996, 1998) marks the $\mathrm{NH}_{3}$ and $\mathrm{N}_{2} \mathrm{H}^{+}$molecules as forming at late
Table 4. Column density ratios.

\begin{tabular}{lccc}
\hline \hline Position & {$\left[\mathrm{HCO}^{+} / \mathrm{CS}\right]$} & {$\left[\mathrm{N}_{2} \mathrm{H}^{+} / \mathrm{CS}\right]$} & $N(\mathrm{CS}) / N_{\max }(\mathrm{CS})$ \\
\hline $\mathrm{S}$ & $<0.21$ & 0.15 & $30 \%$ \\
$\mathrm{E}$ & $<0.07$ & 0.05 & $77 \%$ \\
$\mathrm{~W}$ & 0.10 & $<0.02$ & $100 \%$ \\
$\mathrm{~N}$ & 0.27 & $<0.02$ & $73 \%$ \\
\hline
\end{tabular}

times, when sufficiently high densities are reached or in long lived clumps. Thus, they should be found in the same region. The coincidence between the distribution of the emission of $\mathrm{N}_{2} \mathrm{H}^{+}$observed by the BIMA interferometer and the $\mathrm{NH}_{3}$ emission detected by single-dish observations, and the overall morphological appearance of the $\mathrm{NH}_{3}$ and $\mathrm{N}_{2} \mathrm{H}^{+}$emission seem to support these predictions. Moreover, both interferometric and single-dish emissions are highly concentrated, and are compatible with being originated in the same gas since the emission of $\mathrm{N}_{2} \mathrm{H}^{+}$could be coming from two clumps of size $\sim 0.6 \times 1^{\prime}$ enclosed inside the higher levels of the lower resolution emission of $\mathrm{NH}_{3}$. The $\mathrm{SO}$ molecule was also marked in our model as a late-time molecule. The near coincidence of the clump found in the marginally detected SO emission with the northern clump of the $\mathrm{N}_{2} \mathrm{H}^{+}$emission would support that classification.

Table 4 shows the column density ratios of $\mathrm{N}_{2} \mathrm{H}^{+}$and $\mathrm{HCO}^{+}$over CS for the four selected positions calculated from the column density determinations of Table 3 , and the CS column density relative to the maximum value, found in position W. We observe that $\mathrm{N}_{2} \mathrm{H}^{+}$abundance is $\sim 5-15 \%$ of that of CS for the $\mathrm{S}$ and $\mathrm{E}$ positions, and becomes lower at the $\mathrm{N}$ and $\mathrm{W}$ positions. $\left[\mathrm{HCO}^{+} / \mathrm{CS}\right]$ is $\$ 20 \%$ for the $\mathrm{S}, \mathrm{E}$, and $\mathrm{W}$ positions, and becomes $\sim 30 \%$ at the $\mathrm{N}$ position, while there is a factor 3 variation in the CS column density. We can then differentiate between the $\mathrm{N}$ and $\mathrm{W}$ positions, where $\mathrm{HCO}^{+}$is clearly detected while $\mathrm{N}_{2} \mathrm{H}^{+}$is not, and the $\mathrm{E}$ and $\mathrm{S}$ positions where $\mathrm{N}_{2} \mathrm{H}^{+}$is clearly detected whereas $\mathrm{HCO}^{+}$is not detected.

\subsection{Modeling the chemical evolution}

We used the results of the model explained in Taylor et al. (1998) to compare the predicted abundances of $\mathrm{HCO}^{+}$and $\mathrm{N}_{2} \mathrm{H}^{+}$with respect to $\mathrm{CS}$, with the values we obtained for the column density ratios, and see if some chemical age determination could be made for these positions. Figure 7 shows the predicted fractional abundances for several molecules of interest for values of collapse, $B=1$, freeze-out, $\mathrm{FR}=0.01$, and final density $n_{\mathrm{f}}=5 \times 10^{4} \mathrm{~cm}^{-3}$ (see Taylor et al. 1996, 1998). We also show the molecular abundance relative to CS for the $\mathrm{HCO}^{+}, \mathrm{N}_{2} \mathrm{H}^{+}$, and $\mathrm{NH}_{3}$ molecules.

We found that the abundance $\left[\mathrm{HCO}^{+} / \mathrm{CS}\right]$ can be explained by the model in relatively short times, between $2-3 \times 10^{6}$ years. Thus, a higher $\mathrm{HCO}^{+}$fractional abundance would indicate a more chemically evolved clump. However, this does not seem to agree with the abundance of $\mathrm{N}_{2} \mathrm{H}^{+}$. First, $\mathrm{N}_{2} \mathrm{H}^{+}$reaches high abundances at later times, and one would expect to find $\mathrm{N}_{2} \mathrm{H}^{+}$at more evolved clumps. Second, the model can not explain fractional abundances of $\mathrm{N}_{2} \mathrm{H}^{+}$similar to those of $\mathrm{HCO}^{+}$, and as high as those we found in the $\mathrm{S}$ and $\mathrm{E}$ positions. 

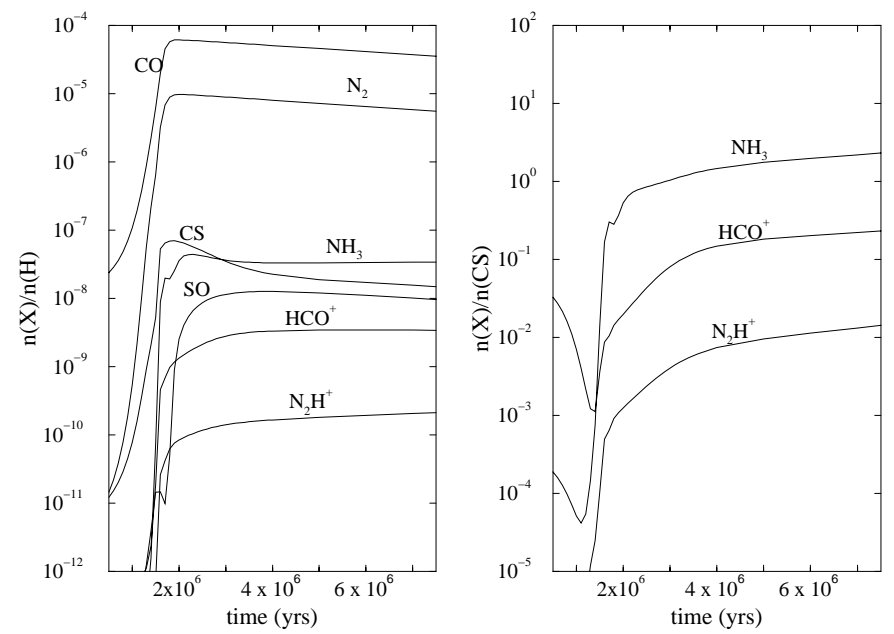

Fig. 7. Left panel: Chemical fractional abundances (relative to $\mathrm{H}$ nuclei) as a function of time for a free-fall collapse model halted at density $n_{\mathrm{H}}=5 \times 10^{4} \mathrm{~cm}^{-3}$. Initially $n_{\mathrm{H}}=1 \times 10^{3} \mathrm{~cm}^{-3}$ and $A_{V}=0.5$. Freeze-out parameter $F R=0.01$, corresponding to an average value of the product of the dust to gas number density ratio and square of the grain radius $\left\langle n_{\mathrm{d}} a^{2}\right\rangle=2 \times 10^{-20} \mathrm{~cm}^{-2}$. Right panel: Fractional abundances of selected species (relative to $\mathrm{CS}$ ) as a function of time.

A slightly higher freeze-out parameter would provide higher relative $\mathrm{N}_{2} \mathrm{H}^{+}$abundance with respect to CS, while not changing appreciably the fractional abundance, because CS is frozenout to grains faster. In this case, we also obtain higher $\mathrm{HCO}^{+}$ relative abundances with CS.

Figure 8 shows the results of the model modifying the final density value to 10 times the previous value, $n_{\mathrm{f}}=5 \times 10^{5} \mathrm{~cm}^{-3}$, and maintaining unchanged the values of $B$ and $F R$. We find some differences with the previous results. CS maximum fractional abundance is similar to the previous model, while $\mathrm{NH}_{3}$, $\mathrm{HCO}^{+}$, and $\mathrm{N}_{2} \mathrm{H}^{+}$reach slightly lower abundances. In any case, $\mathrm{CS}$ and $\mathrm{NH}_{3}$ show the same behavior found in the model of Taylor et al. (1996). However, the higher final density produces a faster depletion of molecules, especially CS which has a steeper decline with time.

At times $\sim 2.5 \times 10^{6}$ years, when $\mathrm{CS}$ has a high fractional abundance, and $\mathrm{HCO}^{+}$has barely reached its maximum fractional abundance, we find a relative abundance $\left[\mathrm{HCO}^{+} / \mathrm{CS}\right] \sim$ 0.1 , while $\mathrm{N}_{2} \mathrm{H}^{+}$has still a low abundance. This could correspond to the $\mathrm{W}$ position of our map. At a later time, $\sim 3 \times$ $10^{6}$ years, when $\mathrm{N}_{2} \mathrm{H}^{+}$is still at low abundances, $\mathrm{HCO}^{+}$abundance is practically the same while CS abundance is slightly lower, with a resulting higher $\left[\mathrm{HCO}^{+} / \mathrm{CS}\right]$ ratio. This could correspond to the $\mathrm{N}$ position. At the time $\mathrm{N}_{2} \mathrm{H}^{+}$reaches its peak abundance, $\sim 4 \times 10^{6}$ years, $\mathrm{HCO}^{+}$has diminished in abundance although it can still be observed. This would point to the $\mathrm{S}$ and $\mathrm{E}$ positions being at later stages of chemical evolution. Distinguishing between the two is difficult. The $\mathrm{S}$ position could be more evolved since it shows a lower CS column density, and a higher $\left[\mathrm{N}_{2} \mathrm{H}^{+} / \mathrm{CS}\right]$ ratio, which could correspond to the stage where $\mathrm{N}_{2} \mathrm{H}^{+}$is at higher abundances.

However, even the modified model is not able to explain the observed $\mathrm{HCO}^{+}$low abundances obtained at positions $\mathrm{S}$ and $\mathrm{E}$ with respect to the predicted values. The $\mathrm{HCO}^{+}$molecule has a
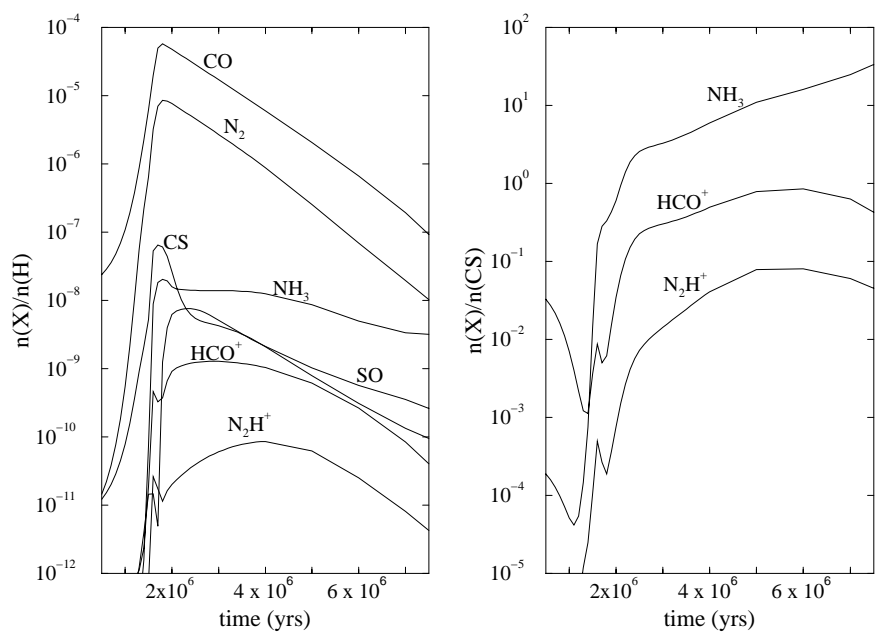

Fig. 8. Left panel: Chemical fractional abundances (relative to $\mathrm{H}$ nuclei) as a function of time for a free-fall collapse model halted at density $n_{\mathrm{H}}=5 \times 10^{5} \mathrm{~cm}^{-3}$. Initially $n_{\mathrm{H}}=1 \times 10^{3} \mathrm{~cm}^{-3}$ and $A_{V}=0.5$. Freeze-out parameter FR $=0.01$, corresponding to an average value of the product of the dust to gas number density ratio and square of the grain radius $\left\langle n_{\mathrm{d}} a^{2}\right\rangle=2 \times 10^{-20} \mathrm{~cm}^{-2}$. Right panel: Fractional abundances of selected species (relative to CS) as a function of time.

high dipole moment, and it has been found that the $(J=1 \rightarrow 0)$ transition line profiles are often self-absorbed in typical molecular cloud cores by foreground gas at lower density and temperature. The apparently sudden lack of $\mathrm{HCO}^{+}$emission in those places where $\mathrm{N}_{2} \mathrm{H}^{+}$or CS emission is intense may suggest that the $\mathrm{HCO}^{+}(J=1 \rightarrow 0)$ emission arising from the core might be absorbed efficiently by a cold low-density envelope around the core or a foreground cloud, as in the case of the NGC 2264G region (Girart et al. 2000).

\subsection{Comparison with other studies}

Studies of chemical evolution have been made up to now with single-dish observations using several transitions of selected early- and late-time molecules, usually $\mathrm{CS}, \mathrm{N}_{2} \mathrm{H}^{+}, \mathrm{NH}_{3}$, $\mathrm{HCO}^{+}, \mathrm{SO}, \mathrm{SO}_{2}$, and $\mathrm{HC}_{3} \mathrm{~N}$. These studies of starless cores show maps where emission seems to originate in several clumps distributed all over the region (Dickens et al. 2000) or in round-like clumps (Tafalla et al. 2002). All these studies show evidences of chemical differentiation. Dickens et al. find that early- and late-time molecules are found at different positions and show differing behavior, i.e. early-time molecules are most abundant where late-time molecules are not, and viceversa. The authors explain their results invoking a difference in the chemical ages of different parts of their region. Tafalla et al. also find that their maps show a great coincidence between the emission of the $\mathrm{N}_{2} \mathrm{H}^{+}$and $\mathrm{NH}_{3}$ molecules, while differing from the CS emission.

Some of these studies also find evidence for differing depletion rates of molecules such as $\mathrm{CS}$ or $\mathrm{NH}_{3}$. Tafalla et al. find a central abundance drop of $\mathrm{CS}$, a constant abundance of $\mathrm{N}_{2} \mathrm{H}^{+}$, and a central enhancement of $\mathrm{NH}_{3}$ in their regions, while Bergin et al. (2001), in observations of the IC 5146 cloud, also detect molecular depletion of $\mathrm{CS}$, while $\mathrm{N}_{2} \mathrm{H}^{+}$remains in 
the gas phase with growing extinction. Other numerical calculations, such as Aikawa et al. (2001) also predict a depletion of certain species from the central regions. In our model, we would expect to find the more massive and chemically older clumps in the central regions. These clumps would have substantial quantities of $\mathrm{NH}_{3}$ and $\mathrm{N}_{2} \mathrm{H}^{+}$, while $\mathrm{CS}$ would have been depleted from the gas phase, which would show as a central depletion of CS.

Thus, these results suggest that although our interferometric observations lose information on the total emission of the region, they are in agreement with the results obtained with single-dish telescopes, suggesting that interferometric observations are useful to study with high angular resolution the chemical evolution of inner structures of starless cores.

\section{Summary}

We made a multitransitional study with the BIMA interferometric array of the starless core found in the L673 (Morata et al. 1997), in order to test the chemical model of Taylor et al. (1996, 1998). The main results were:

1. We detected emission in the $\mathrm{CS}(J=2 \rightarrow 1), \mathrm{N}_{2} \mathrm{H}^{+}$ $(J=1 \rightarrow 0)$, and $\mathrm{HCO}^{+}(J=1 \rightarrow 0)$ lines. We marginally detected emission in the $\mathrm{SO}\left(J_{K}=2_{3} \rightarrow 1_{2}\right)$ line.

2. The high angular resolution interferometric observations revealed a much clumpier medium than the lower resolution single-dish observations. Several clumps of size $\lesssim 0.08 \mathrm{pc}$ were found for each line distributed all over the region where the single-dish emission was found.

3. Each molecular transition traced differently the clump distribution, although in some cases the detected clumps were coincident. We found that the distribution of the $\mathrm{N}_{2} \mathrm{H}^{+}$ emission was completely enclosed by the half-maximum contours of the $\mathrm{NH}_{3}$ maps of Sepúlveda et al. (2002), and that both emissions were highly compact and compatible with being originated in the same gas. We also found a marginal coincidence with the SO molecule, which is also a late-time molecule.

4. The BIMA interferometer detected only $9-12 \%$ of the CS $(2 \rightarrow 1)$ emission. Modelling the filtering effect of BIMA, we found that a clumpy and heterogeneous medium could explain this effect.

5. We estimated the abundance ratios $\left[\mathrm{N}_{2} \mathrm{H}^{+} / \mathrm{CS}\right]$ and $\left[\mathrm{HCO}^{+} / \mathrm{CS}\right]$ from the molecular column density determinations in four selected positions, labeled as $\mathrm{S}, \mathrm{E}, \mathrm{W}$, and $\mathrm{N}$, in order to compare them with the predicted values of the chemical model. We found that at the $\mathrm{N}$ and $\mathrm{W}$ positions there was a high $\mathrm{HCO}^{+}$abundance relative to $\mathrm{CS}$ and a low $\mathrm{N}_{2} \mathrm{H}^{+}$abundance, while at the $\mathrm{S}$ and $\mathrm{E}$ positions the case was reversed.

6. The chemical model was able to explain the abundance ratios of $\left[\mathrm{HCO}^{+} / \mathrm{CS}\right]$ at the positions where $\mathrm{HCO}^{+}$was detected, $\mathrm{N}$ and $\mathrm{W}$. The best fit was for a model with a density at which collapse is halted of $n_{\mathrm{f}}=5 \times 10^{5} \mathrm{~cm}^{-3}$. In this case, we found that the predicted $\left[\mathrm{N}_{2} \mathrm{H}^{+} / \mathrm{CS}\right]$ abundance ratios were more in agreement with the observations. A high $\mathrm{HCO}^{+}$abundance, but a low $\mathrm{N}_{2} \mathrm{H}^{+}$abundance would represent an earlier stage, where the $\mathrm{W}$ and $\mathrm{N}$ positions could be found. The $\mathrm{S}$ and $\mathrm{E}$ positions would be in a later chemical stage on account of the higher $\mathrm{N}_{2} \mathrm{H}^{+}$abundance, although a more precise differentiation is hard to make.

Thus, the model described in Taylor et al. $(1996,1998)$ applied to a starless core with no apparent signs of other star formation tracers, such as molecular outflows or radio continuum sources, was able to explain the interferometric observations, if we take into account the likely self-absorption of $\mathrm{HCO}^{+}$. In particular, the chemical differentiation of the detected clumps and the coincidence of the $\mathrm{NH}_{3}$ and $\mathrm{N}_{2} \mathrm{H}^{+}$emissions agree with the model. It also enabled us to explain the $\mathrm{HCO}^{+}$and $\mathrm{N}_{2} \mathrm{H}^{+}$ emissions and to propose a classification of the studied clumps according to the stage of chemical evolution indicated by the molecular abundances.

Further multitransitional observations with higher sensitivity would be necessary in order to determine with more detail the physical structure of the region and to provide more data with which to refine the chemical model predictions. In particular, the observation of molecules such as $\mathrm{H}_{2} \mathrm{CO}$ and $\mathrm{SO}$, or of the isotope $\mathrm{H}^{13} \mathrm{CO}^{+}$, would help to clarify the nature of the $\mathrm{HCO}^{+}(J=1 \rightarrow 0)$ emission.

Acknowledgements. JMG acknowledges support by NSF grant AST99-81363 and by RED-2000 from the Generalitat de Catalunya. RE and JMG are partially supported by DGICYT grant PB98-0670 (Spain). We thank the referee for the comments and discussion.

\section{References}

Aikawa, Y., Ohashi, N., Inutsuka, S.-I., Herbst, E., \& Takakuwa, S. 2001, ApJ, 552, 639

Beichman, C. A., Myers, P. C., Emerson, J. P., et al. 1986, ApJ, 307, 337

Benson, P. J., \& Myers, P. C. 1989, ApJSS, 71, 89

Bergin, E. A., Ciardi, D. R., Lada, C. J., Alves, J., \& Lada, E. A. 2001, ApJ, 557, 209

Caselli, R., Myers, P. C., \& Thaddeus, P. 1995, ApJ, 455, L77

Dickens, J. E., Irvine, W. M., Snell, R. L., et al. 2000, ApJ, 542, 870

Girart, J. M., \& Acord, J. M. P. 2001, ApJ, 552, L63

Girart, J. M., Estalella, R., Ho, P. T. P., \& Rudolph, A. L. 2000, ApJ, 539,763

Herbig, G. H., \& Jones, B. F. 1983, AJ, 88, 1040

Morata, O., Estalella, R., López, R., \& Planesas, P. 1997, MNRAS, 292,120

Myers, P. C., Fuller, G. A., Goodman, A. A., \& Benson, P. J. 1991, ApJ, 376, 561

Pastor, J., Estalella, R., López, R., et al. 1991, A\&A, 252, 320

Pauls, T. A., Wilson, T. L., Bieging, J. H., \& Martin, R. N. 1983, A\&A, 123,23

Sault, R. J., Teuben, P. J., \& Wright, M. C. H. 1995, in Astronomical Data Analysis Software and Systems IV, ed. R. A. Shaw, H. E. Payne, \& J. J. Hayes (San Francisco: Astronomical Society of the Pacific), ASP Conf. Ser. 77, 433

Sepúlveda, I., et al. 2002, in preparation

Tafalla, M., Myers, P. C., Caselli, P., Walmsley, C. M., \& Comito, C. 2002, ApJ, 569, 815

Taylor, S. D., Morata, O., \& Williams, D. A. 1996, A\&A, 313, 269

Taylor, S. D., Morata, O., \& Williams, D. A. 1998, A\&A, 336, 309

Wright, M. C. H. 1996, BIMA memo 45

Zhou, S., Wu, Y., Evans, N. J., Fuller, G. A., \& Myers, P. C. 1989, ApJ, 346, 168 